\title{
Preparación de un Artículo para ser Publicado en la Revista Internacional Formación Universitaria
}

\author{
José 0. Valderrama ${ }^{1,2}$ \\ (1) Fac. de Ingeniería, Depto. Ing. Mecánica, Univ. de la Serena, Casilla 554, La Serena-Chile \\ (2) Centro de Información Tecnológica (CIT), Casilla 724, La Serena-Chile
}

\begin{abstract}
Resumen
Se presentan algunas reflexiones y recomendaciones sobre como se debe organizar, estructurar y presentar un trabajo que desea ser considerado para publicación en la revista Formación Universitaria u otras revistas similares en alcances y contenidos. Este artículo resume algunos conceptos vertidos por el autor en un artículo anterior y discute aspectos generales sobre trabajos que no necesariamente están basados en investigación científica y que corresponden a lo que se denomina reflexión documentada. Este tipo de artículos es aceptable en la revista Formación Universitaria pero deben incluir documentación adecuada y similar a la exigida en otras revistas de corriente principal. Esto porque la documentación, en especial con artículos de revistas de corriente principal, es la que le da el soporte "científico" al artículo. Se concluye sobre la necesidad de que los autores cuiden que sus escritos se ajusten a ciertas normas mínimas de forma y de fondo aceptadas por la comunidad internacional si desean que sus artículos, una vez publicados, tengan el impacto deseado.
\end{abstract}

Palabras clave: formación universitaria, publicaciones, manuscritos, organización de un artículo, congresos y publicaciones

\section{Preparation of a Manuscript to be Published in the International Journal Formación Universitaria}

\begin{abstract}
Some reflections and recommendations on how to organize, to structure and to submit a manuscript to be considered for publication in the journal Formación Universitaria or other journals with similar scope and subjects are presented. This papers summarizes some concepts presented by the author in a previous communication and discusses some general aspects on articles that are not necessarily based on scientific research but belong to the so-called documented reflection. This type of articles is acceptable in Formación Universitaria but it is required to include appropriate documentation, similar to that requested in any other main stream journal. This is because it is the documentation that gives the "scientific" support to the article. It is concluded ion the need that authors must follow the instructions for authors established by the journal and by the international community if they want that their papers have good impact once they are published.
\end{abstract}

Keywords: university formation, publication, manuscript, paper organization, journals and congresses 


\section{INTRODUCCIÓN}

En un artículo del año 2005 publicado en la revista Información Tecnológica, el autor describe los principales elementos que se debe considerar al escribir y preparar un artículo para una revista internacional de corriente principal (Valderrama, 2005). Información Tecnológica es una revista internacional arbitrada indizada y en idioma Castellano, considerada en Chile y en otros países como una revista "de corriente principal" que publica trabajos originales en el amplio campo de la ciencia, la ingeniería y la tecnología, y es publicada desde el año 1990 por el Centro de Información Tecnológica (CIT), de La Serena en Chile (www.citchile.cl).

El año 2008, el CIT empieza la publicación de Formación Universitaria, una revista internacional electrónica y arbitrada que publica artículos originales en todas las áreas relacionadas con el trabajo académico en Universidades, y en particular aquellos relacionados con la enseñanza y formación universitaria, con énfasis en las ingenierías y las ciencias, pero no restringidos a ellas. Desde sus inicios, la revista estableció que es condición obligatoria que los artículos sean producto de trabajos de investigación o de reflexión documentada y que tengan un impacto relevante en el desarrollo y mejoramiento de la formación universitaria en Ibero América (http://www.citchile.cl/a1-formacion.htm).

De estas definiciones, lo que la revista describe como "reflexión documentada" requiere comentarios y aclaraciones ya que por ignorancia o conveniencia no es bien interpretada. A esta categoría corresponden artículos relacionados con experiencias en aulas, implementación de nuevos laboratorios, metodologías de enseñanza-aprendizaje, modificación y propuestas de planes de estudios y programas, experiencias en acreditación, jerarquización, y gestión universitaria, entre muchos otros. En estos temas la revista no solo acepta trabajos basados en investigación científica sino también ideas y reflexiones sobre dichos tópicos, experiencias personales en la enseñanza o actividades de gestión académica, Sin embargo, todos los artículos deben ser necesariamente presentados como trabajo documentado y estructurado como se hace en una revista de corriente principal. Para aclarar aún más las ideas, la Tabla 1 lista una serie de artículos publicados en Formación Universitaria en los últimos dos años y que dan una idea más completa del tipo de artículos aceptables. Otros títulos pueden ser vistos en la propia Web de la revista (http://www.citchile.cl/a1-formacion.htm).

Como se sabe, la publicación en revistas arbitradas, indizadas y de alto impacto en la comunidad científica y tecnológica, llamadas también "de corriente principal", tienen un alcance mucho mayor que cualquier otro medio de difusión de un trabajo de investigación (Plomp, 1994; Amin y Cabe, 2000; Packer, 2003). Esta es una de las principales razones para considerar a la publicación en revistas arbitradas, como un recurso estratégico de la investigación y de la difusión del trabajo académico en una universidad (Valderrama, 2001). Si un investigador publica, entonces se da a conocer, sus trabajos son citados y dan origen a nuevos estudios, puede optar a dineros para proyectos, puede elevar el nivel de sus investigaciones, y obviamente prestigiar a la institución a la que pertenece. Además, en el caso de investigadores universitarios la investigación y su difusión a la comunidad internacional representan importantes apoyos para la formación de los futuros profesionales. La revista Formación Universitaria representa un medio de amplia difusión para compartir ideas y reflexiones en torno a la actividad académica de enseñanza-aprendizaje, gestión universitaria y todo aspecto relacionado con la formación universitaria de profesionales.

Conozco muchos académicos dedicados a la importante tarea de formar profesionales que creen que basta solo con transmitir conocimiento en la forma que lo hicieron con ellos o ellas o bien usando lo que, desde su muy limitado punto de vista, consideran adecuado como método de enseñanza. Hay muchos otros, sin embargo que están en continuo aprendizaje y experimentando formas nueva de avanzar, de acuerdo al ritmo del resto de la sociedad, de las tecnologías de la información y de otros avances que tienen ingerencia en el mundo universitario. Pero cuando se trata de transmitir esas experiencias al resto de la comunidad académica a través de documentos escritos como son las publicaciones en revistas, se observan problemas graves que es preciso comentar, analizar y dar recomendaciones para su solución. Uno de los principales problemas que enfrentan muchos autores iberoamericanos, en especial aquellos de poca experiencia en investigación y publicaciones, es la forma en que deben ser presentadas las diversas partes que constituyen la estructura de un artículo, tanto en asuntos de forma como de contenido. 
Tabla 1: Selección de títulos de artículos publicados en Formación Universitaria (http://www.citchile.cl/a1-formacion.htm).

\begin{tabular}{|l|}
\hline Aplicación del Simulador ChemCAD en la Enseñanza en Carreras de Ingeniería \\
\hline Innovación Pedagógica y Elaboración de una Guía de Aprendizaje en Tecnología Farmacéutica Industrial \\
\hline Expresión de Conceptos Químicos Mediante Lenguaje Significativo \\
\hline Análisis de Hábitos de Estudio en Alumnos de Primer Año de Ingeniería Civil Agrícola \\
\hline Gestión de la Calidad y del Medio Ambiente en Instituciones de Educación Superior \\
\hline Colaboración Académica Internacional en Tecnologías de la Información y Docencia Virtual \\
\hline Resolución de Problemas Integradores en la Enseñanza de la Física para Estudiantes de Ingeniería Civil \\
\hline Equipo Didáctico para Aprendizaje Colaborativo en Automatización e Informática Industrial. \\
\hline Como Medir y Guiar Cambios hacia Entornos Educativos más Motivadores \\
\hline Evaluación del Proceso de Enseñanza-Aprendizaje sobre Fuentes Renovables de Energía \\
\hline Enseñanza-Aprendizaje en Robótica. Construcción de Simuladores como Actividades de Comprensión. \\
\hline Nuevo Perfil para el Farmacéutico: la Readaptación de los Currículos en Cursos de Farmacia \\
\hline Sistema Europeo de Transferencia de Créditos (ECTS) en una Asignatura de Química para Ingeniería \\
\hline Evaluación de las Tecnologías de Información en la Docencia de Proyectos de Ingeniería \\
\hline Aprender Enseñando: Elaboración de Materiales Didácticos que facilitan el Aprendizaje Autónomo \\
\hline Programa Emprendedor Obligatorio. Alternativa de Educación Integral \\
\hline Educación Superior Virtual en América Latina: Perspectiva Tecnológica-Empresarial. \\
\hline Qué Piensan los Profesores sobre sus Clases: Creencias Curriculares y Creencias de Actuación Curricular \\
\hline
\end{tabular}

En el artículo ya mencionado del autor (Valderrama, 2005), se exponen algunas ideas, reflexiones, experiencias y recomendaciones relacionadas con la preparación de un artículo para ser enviado a una revista de corriente principal. Los conceptos, ideas y sugerencias vertidos en ese trabajo (http://www.scielo.cl/scielo.php?script=sci_arttext\&pid=S0718-07642005000200002\&/ng=es\&nrm=iso\&tlng= es), fueron recolectados de la literatura (Bishop, 1984; Day, 1983; King, 2004; Campanario, 2009; CIT, 2010), y de la propia experiencia del autor como Editor, árbitro de numerosas revistas de corriente principal, revisor de proyectos internacionales, y miembro del comité Editorial de varias revistas El autor expone allí detalles sobre como estructurar el título, el resumen, las palabras clave y todas las demás secciones de un articulo. Todo lo vertido allí está más bien orientado a trabajos derivados de investigación científica en la ingeniería y las ciencias, pero prácticamente todo lo presentado es igualmente válido para trabajos de divulgación de experiencias docentes y reflexiones sobre todos los aspectos de la formación de nuestros estudiantes, tema central de la revista Formación Universitaria.

En este trabajo se enfatiza algunos aspectos que seguro serán de especial interés para autores iberoamericanos que se dedican probablemente a enseñar y fomentar la buena docencia, hacer experiencias en aulas y desarrollar metodologías novedosas en el campo de la enseñanza-aprendizaje, más que a la investigación científica pura. También serán de interés para los administradores académicos de las universidades que han dedicado parte de su carrera universitaria a la gestión más que a la difusión de trabajos de investigación y reflexión académica. Por lo tanto, se recomienda a quienes deseen publicar en Formación Universitaria leer y analizar todos los aspectos del trabajo anterior (Valderrama, 2005), que sin duda representa un complemento indispensable a lo presentado en este artículo.

\section{REFLEXIÓN DOCUMENTADA}

Este término se refiere a un escrito donde se analiza, reflexiona, discute y propone ideas referentes a algún tema sobre el cual no se ha hecho un trabajo científico metódico y donde muchas veces no existe información "dura" como son los números en un experimento en química, física, matemáticas o ingeniería. Esta falta de información cuantitativa es al parecer la razón de porqué autores de este tipo de trabajos creen que artículos de reflexión o ideas nuevas que ellos o ellas plantean en sus 
artículos no necesitan ser respaldadas por trabajos de otros y escriben como si nadie más hubiera presentado algo sobre el tema que tratan. Este es sin duda el principal problema de fondo en los manuscritos recibidos, y que muchas veces es, con justa razón, causal de rechazo de los trabajos enviados para publicación.

La documentación que respalda el trabajo que se presenta en un artículo va en la primera sección, la Introducción, aunque hay revistas que la incluyen en otras secciones. Independiente de su ubicación y del nombre que se le de a la sección el objetivo de esta parte del escrito es proporcionar al lector(a) antecedentes suficientes que le permitan ubicar el tema y el artículo en el contexto de la literatura pasada y presente. El autor debe escoger cuidadosamente la literatura a citar en esta sección y aclarar porqué, existiendo toda esa información, el artículo que se presenta es necesario. En esta sección se debe: i) especificar la naturaleza y alcance del problema tratado; ii) presentar una revisión de la literatura sobre el tema del artículo; iii) analizar brevemente esos trabajos; iv) detallar la propuesta del artículo; y v) concluir respecto al estudio, análisis o reflexión.

En la revisión de la literatura pasada y presente se debe dar especial importancia a la literatura de los últimos años, sin olvidar clásicos del pasado que el autor considere apropiado incluir. Lo que no puede pasar, pero que ocurre con cierta frecuencia, es citar solamente literatura de hace 30040 años, como si nadie hubiera trabajado en el tema en las últimas décadas. Es también poco adecuado y poco conducente incluir autocitas en exceso o solamente trabajos disponibles en la llamada literatura gris, como si eso fuera todo el conocimiento disponible en el tema tratado en el artículo. El conocimiento establecido y actual es usualmente el presentado en las revistas de corriente principal o en libros seleccionados y reconocidos por todos como fuentes válidas de conocimiento.

En trabajos poco o mal documentados uno podría tener legítimas dudas respecto a por ejemplo la originalidad de las ideas y propuestas: “ ¿como saben los autores que lo que presentan no ha sido ya publicado si no revisaron la literatura?". La inclusión de citas pertinentes, adecuadas y oportunas en un escrito permite al autor ubicar el trabajo que presenta en un contexto universal y actual del conocimiento en el tema específico del artículo, y permite al lector documentarse sobre avances previos en ese campo y que sustentan el trabajo actual. No revisar la literatura actual e incorporarla adecuadamente en un artículo es pasar por alto información útil y esencial para construir una idea, una teoría, un método o una aplicación en particular. Hoy en día, con una tremenda biblioteca prácticamente "gratis" como es Internet, es simplemente inaceptable que un autor no verifique si sus ideas, plasmadas en el trabajo que presenta, no fueron ya ideadas por otros y publicadas en otra parte.

La cantidad de citas y referencias no está establecida y es casi imposible hacerlo. Sin embargo, hay una norma general que debe prevalecer sobre cualquier otro concepto: hay que incluir las que sean necesarias. Para los autores con cierta experiencia esto es fácil de determinar y de hacer, asunto que no es tan claro para quienes tienen menos experiencia en estos asuntos de las publicaciones internacionales. Las citas necesarias serán aquellas que permitan al lector documentarse en forma general sobre lo hecho en el pasado en el tema del artículo y que le permita verificar las teorías e hipótesis que sustentan el trabajo que lee. Un articulo tipo revisión (review) requiere obviamente de más citas y referencias que un articulo de investigación sobre un tema o experiencia concreta. Con respecto al tipo de literatura citable, tampoco hay normas establecidas, pero claramente son los artículos publicados en revistas de corriente principal donde están presentados los avances en diversas áreas de la ciencia, la ingeniería y las humanidades, y estos artículos son los que deben prevalecer en una publicación. Esto no significa que no se pueda citar otras fuentes como manuales, enciclopedias, libros, tesis, documentos de empresas, catálogos y cualquier otro documento. Lo que es inaceptable, como ocurre a veces es que un artículo base todo su nuevo aporte en un par de tesis que nadie validó o en unas páginas abiertas en Internet a veces de dudosa procedencia. Otras veces los autores basan sus artículos en sus propios trabajos anteriores que nunca publicaron, y se autocitan con informes internos de muy difícil acceso para un lector del mundo.

Finalmente, se debe comentar un aspecto más de forma pero igualmente importante y que está relacionado con la forma de detallar un documento en el listado de referencias (artículos, libros, monografías, páginas en Internet, entre otros). Aunque hay normas que establecen las revistas, muchas veces debe primar el sentido común. Si un artículo tiene muchos autores, por ejemplo 25, 
de los cuales Araya es el primero, entonces parece poco racional listar los nombres de todos los autores en el listado de Referencias. En este caso se puede colocar, Araya y otros 24 autores. Esto es de especial importancia ya que algunas revistas no limitan el número de autores y ocurren situaciones poco comprensibles. Si 25 autores puede parecer mucho, que más decir sobre un artículo en Physical Review Letters con 411 autores de 58 instituciones (Adare et al., 2007)

\section{TRANSFORMAR COMUNICACIONES A CONGRESOS EN PUBLICACIONES}

Muchos de los trabajos enviados para publicación en revistas provienen de comunicaciones que recientemente los autores han presentado en congresos, simposios y conferencias. Este asunto no es cuestionable desde ningún punto de vista. La presentación de un trabajo en un congreso y su posterior publicación como artículo acabado y completo en una revista internacional es la culminación natural y óptima de una buena investigación, sin desestimar la publicación de monografías para trabajos de mayor envergadura. Lo que es cuestionable y no aceptable es enviar exactamente el mismo trabajo del congreso a una revista.

Las presentaciones en congresos, seminarios, conferencias y eventos similares, no constituyen publicación y no debieran ser nunca confundidos. Estas actividades constituyen sin duda importantes instancias de encuentro para que profesionales e investigadores puedan intercambiar ideas y compartir experiencias, además de reflexionar sobre diversos aspectos relacionados con el tema del evento. Por cierto que es bueno que existan y que sigan existiendo en el futuro, pero desde el punto de impacto en la comunidad docente e investigadora, no vale lo mismo una presentación en un congreso y su impresión en las Actas, por muy relevante que sea el congreso, y una publicación en una revista internacional de corriente principal. Es en la publicación en revistas donde se validan los resultados de una investigación. Las Actas de Congresos (Proceedings), representan obras escritas de importancia para la difusión de trabajos de investigación en marcha y en la mayoría de los casos constituyen una muestra más o menos fidedigna del estado de la investigación en un determinado tema. Los trabajos incluidos pueden haber sido evaluados por pares y la obra puede tener ISBN y Registro de Propiedad Intelectual, pero siguen siendo Actas de Congresos y no publicaciones.

Algunas diferencias fundamentales sobre una publicación y una presentación en un congreso son: i) en un congreso un trabajo no debe estar necesariamente acabado, en una publicación si; ii) una publicación debe ser necesariamente arbitrada por pares en forma rigurosa, en un congreso esto no es necesariamente cierto; iii) un trabajo presentado en un congreso tiene un alcance reducido, los congresales y otros pocos lectores de las limitadas Actas, mientras que una publicación puede ser leída por miles de lectores; iv) las publicaciones constituyen información final para sustentar investigación futura, mientras que para un trabajo de congreso esto no es un requisito; y v) un trabajo de congreso no necesita estar apropiadamente documentado, mientras que en una publicación, la documentación es obligatoria. Este último punto es uno de los más comúnmente ignorados y confundidos por los autores que envían lo que fueron sus comunicaciones a congresos sin cambio alguno para ser publicados en una revista de corriente principal. Esto es definitivamente no aceptable. Además de asuntos de fondo que es necesario introducir para transformar una presentación en congreso en una publicación, se observa con frecuencia una cierta resistencia de algunos autores y autoras a seguir normas establecidas por los editores y publicadores. No leer las normas de publicación que imponen las revistas internacionales para los artículos, interpretarlas en forma errónea pero conveniente o simplemente no cumplirlas, es una situación más común que lo deseable o imaginable. Aparte de tener algo que difundir a la comunidad internacional, hay cuatro factores principales en las Normas de una revista y que muchas veces determinan que un manuscrito sea o no publicable: i) contenido; ii) estructura del artículo; iii) ortografía y redacción; y iv) normas de edición. Los aspectos de contenido se refieren a documentación adecuada, rigurosidad de la metodología, credibilidad de los resultados, precisión de la discusión, validez de las conclusiones, inclusión de citas adecuadas y exactitud de las referencias. Varios de estos aspectos han sido discutidos previamente en la literatura (Day, 1983; Valderrama, 2005; Campanario, 2009; CIT, 2010). La organización del trabajo es también importante y siempre vienen explicadas en las normas que cada revista establece. La figura 1 muestra la pantalla principal con enlaces de interés para autores $y$ lectores $y$ las Normas de la revista se encuentran en http://www.citchile.cl/informacion-autores-formacion.htm 


\section{CiT Formación Universitaria}

\begin{tabular}{|c|c|}
\hline $\begin{array}{l}\text { Comité Editorial } \\
\text { (Editor, Comité y Publicadores) }\end{array}$ & $\begin{array}{l}\text { Formación Universitaria es una revista internacional } \\
\text { electrónica y arbitrada, y acepta artículos originales en } \\
\text { todas las áreas relacionadas con el trabajo académico }\end{array}$ \\
\hline $\begin{array}{l}\text { Normas de Publicación } \\
\text { (como preparar y enviar articulos) }\end{array}$ & $\begin{array}{l}\text { en Universidades, y en particular aquellos relacionados } \\
\text { con la enseñanza de la ingeniería y las ciencias. Es } \\
\text { condición obligatoria que los artículos sean producto de }\end{array}$ \\
\hline $\begin{array}{l}\text { Sistema de Arbitraje } \\
\text { (proceso y documentos) }\end{array}$ & $\begin{array}{l}\text { trabajos de investigación o de reflexión documentada } \\
\text { que tengan un impacto relevante en el desarrollo } \\
\text { mejoramiento de la formación universitaria en lbe }\end{array}$ \\
\hline $\begin{array}{l}\text { Acerca de la Revista } \\
\text { (Indices donde está incluida la revista) }\end{array}$ & $\begin{array}{l}\text { revista buscará posicionarse en el ambiente } \\
\text { iberoamericano para ser reconocida en el corto plazo } \\
\text { como un medio de publicación válido para artículos }\end{array}$ \\
\hline $\begin{array}{l}\text { Acceso a Artículos } \\
\text { (bajar articulos y como citarios) }\end{array}$ & $\begin{array}{l}\text { derivados de proyectos que reciben apoyo universita } \\
\text { qubernamental o de instituciones internacionales. }\end{array}$ \\
\hline
\end{tabular}

Comité Editorial

Normas de Publicación

(bajar articulos y como citarios)

\begin{abstract}
Formación Universitaria es una revista internacional electrónica y arbitrada, y acepta artículos originales en todas las áreas relacionadas con el trabajo académico en Universidades, y en particular aquellos relacionados condición obligatoria que los articulos sean producto de trabajos de investigación o de reflexión documentada y que tengan un impacto relevante en el desarrollo y mejoramiento de la formación universitaria en lbero revista buscará posicionarse en el ambiente como un medio de publicación válido para artículos gubernamental o de instituciones internacionales.
\end{abstract}

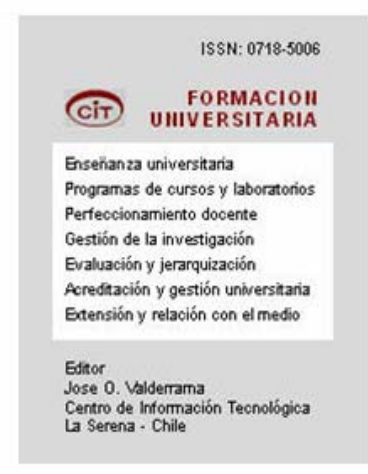

Congreso CAIP Otros Servicios is

Fig. 1: La página Web de la revista Formación Universitaria y sus principales enlaces, incluidas las Normas de Publicación (http://www.citchile.cl/a1-formacion.htm).

Hay también asuntos de política editorial que obligan a los autores a seguir cierta estructura en el artículo y que puede ser diferente en diferentes revistas. En ortografía y redacción los problemas más frecuentes son: puntuación incorrecta, uso excesivo de párrafos, cambio de forma y tiempo verbal, uso de barbarismos y anglicismos, mal uso de mayúsculas y enumeración excesiva de secciones. En Normas de edición hay varias faltas frecuentes: márgenes, espaciado, tipo de letra, sangrías, separación de párrafos, estructura de tablas y figuras, uso de letras en negrita o en cursiva y subrayado, entre otros. Las Normas en cuanto a márgenes y espaciado o a la estructura y presentación de tablas y figuras no es una cuestión menor. Hay asuntos de tipo editorial que obligan a mantener ciertos formatos en dimensiones del papel y de los márgenes. Pero más importante es que el respeto que un autor o autora tenga por estos aspectos refleja su capacidad para ajustarse a ciertos requisitos y normas, que son igualmente pedidos en los protocolos experimentales, en la formulación de modelos, en el desarrollo de software, análisis de encuestas, o simplemente en las reflexiones que exponen.

\section{LOS ERRORES MÁS COMUNES EN TRABAJOS ENVIADOS PARA PUBLICACIÓN}

Aunque los errores que cometen los autores en los trabajos que envían para publicación a una revista o para presentación en un congreso son muy diversos, dependiendo principalmente de la experiencia de los autores, hay algunos comentarios y reflexiones generales que creo pueden ser útiles para los autores novatos y porqué no también para los más experimentados. Entre las faltas más comunes están: mala estructuración del resumen, pobre discusión de los resultados y mala redacción de las conclusiones. Pero probablemente la más grave es la falta de documentación en la introducción, tema que ha sido analizado mas arriba. Sobre los otros aspectos, igualmente importantes para la correcta preparación de un artículo, se comenta en lo que sigue.

El punto común en todas las formas de difusión de los resultados de una investigación (tesis, monografías, informes, comunicaciones a congresos, publicaciones), es que hay revisores y editores a quienes convencer con nuestros escritos, hay lectores a quienes satisfacer y cautivar con 
nuestras ideas, y hay una comunidad científica que siempre nos juzga por la forma en que escribimos. Si todo esto no motiva a un autor para presentar un buen trabajo siguiendo lineamientos internacionales establecidos, es mejor que no publique. Para tener éxito en el tramite de evaluación y aceptación final de un trabajo enviado a una revista es fundamental, antes de todo, seguir las Normas que establece dicha revista. Si los autores o autoras no son capaces de seguir las normas de formato establecidas para la confección de un artículo, ¿porqué debo pensar que siguieron normas en sus experimentos y que sus resultados son correctos?". Los autores novatos que probablemente no lo saben y los experimentados que a veces lo olvidan, deben preocuparse que sus escritos sigan dichas normas, que para eso fueron escritas. Las recomendaciones usuales sobre tres secciones importantes, aparte de la Introducción ya discutida más arriba, como son el Resumen, la Discusión y las Conclusiones se condensan en lo que sigue.

Un resumen debe contener lo que se presenta en el artículo, en un lenguaje simple y directo. El Resumen debe, en este mismo orden: (i) establecer el objetivo y alcance del estudio presentado en el artículo; (ii) entregar antecedentes y describir la metodología; (iii) resumir los resultados más importantes; y (iv) establecer las principales conclusiones. La sección de Discusión constituye una parte esencial en la que se debe analizar los resultados, discutir sus implicancias y resaltar el alcance de ellos. Muchos artículos, que pueden ser atractivos por su temática, por su revisión de la literatura, por su metodología científica, y por sus interesantes y válidos resultados, son rechazados o tienen poco impacto por una mala discusión. La sección de Conclusiones constituye una parte esencial y obligatoria para cerrar un artículo. En esta sección se debe concluir en forma directa y simple sobre lo encontrado en la investigación descrita; no se debe discutir nada (eso va en la sección de Discusión), no se debe recapitular el trabajo en forma condensada (eso va en la sección Resumen), ni se debe presentar resultados (eso va en la sección Resultados). Una forma clásica y adecuada, y que muchos autores(as) prefieren, es escribir una frase de presentación y luego mostrar las conclusiones numeradas.

De todos estos elementos el resumen tiene especial impacto en la comunidad lectora e investigadora, por varias razones. Se debe mencionar que las primeras impresiones que se forma un árbitro al evaluar un artículo estarán basadas en el Título y en el Resumen, y puede ocurrir que un árbitro se forme una idea errónea de un artículo por la sola lectura de un Resumen mal escrito. A menudo se cumple también lo que claramente expresa Day (1983) sobre este asunto: "un buen Resumen viene seguido de un buen artículo; un Resumen pobre es el preámbulo de inconsistencias y errores". Una vez que se tiene buen material para publicar, muchos de los errores comentados más arriba y sus consecuencias (discusión con los editores, atrasos en la publicación y los malos ratos que ello conlleva), pueden ser evitados con una simple regla: debo leer las instrucciones de presentación o normas establecidas por la revista y seguirlas en todos los aspectos. Como autor no dude que si el trabajo es aceptado por los pares evaluadores, un buen editor siempre ayudará a que le trabajo sea mejor presentado y tenga mejor impacto en la comunidad a la que está dirigido el artículo. En la revista Formación Universitaria los autores tienen incluso un listado de revisión (checklist) con el que pueden revisar los principales asuntos de forma y de fondo que son requeridos para iniciar el proceso de evaluación y edición de un artículo. La Tabla 2 muestra un extracto de dicha lista.

Tabla 2: Listado de revisión para ser usado antes de enviar un artículo a publicación. El listado completo está en http://www.citchile.cl/documentos/tabla-de-revision-trabajo-original-fu.pdf

\begin{tabular}{|l|l|}
\hline Aspectos de forma a comprobar & $\begin{array}{l}\text { Marque } \\
\text { X }\end{array}$ \\
\hline Las páginas tienen el tamaño correcto (oficio de $8.5 \times 13$ pulgadas $=21.6 \times 35.6 \mathrm{~cm}$ ) & \\
\hline Todos los párrafos están ajustados a ambos lados (justified) con separación simple entre párrafos & \\
\hline Todas las ecuaciones están numeradas & \\
\hline No hay numeración de secciones ni subsecciones & \\
\hline No hay ningún pie de páginas (en texto, figuras y tablas) & \\
\hline Todas las figuras son editables (que el editor las pueda editar, si es necesario) & \\
\hline
\end{tabular}




\begin{tabular}{|l|l|}
\hline Aspectos de fondo a comprobar & $\begin{array}{l}\text { Marque } \\
\text { X }\end{array}$ \\
\hline El Resumen no supera las 15 líneas y sigue la estructura indicada en las Normas de la Revista & \\
\hline La Introducción está bien documentada (suficientes citas a la literatura) & \\
\hline El trabajo incluye una sección corta de "Conclusiones" & \\
\hline Las citas a la literatura están por autor y año (Arias, 2007; Pérez y Rojas, 2006; Pérez et al., 2005) & \\
\hline Las referencias están completas, como para poder llegar a ellas en forma inequívoca & \\
\hline Toda cita aparece en el listado de Referencias y toda referencia aparece citada en el trabajo & \\
\hline
\end{tabular}

\section{CONCLUSIONES}

De los argumentos presentados, de su discusión y de los antecedentes de la literatura expuestos a través del artículo, se pueden obtener las siguientes conclusiones principales:

1.- artículos de exposición de ideas y reflexiones deben incluir documentación adecuada y similar a la exigida en otras revistas de corriente principal;

2.- las citas de la literatura en una publicación, documentan los argumentos, ideas, conceptos, teorías y datos usados y expuestos en el escrito, y por lo tanto deben incluir trabajos recientemente publicados

3.- la documentación, en especial con artículos de revistas de corriente principal, es la que le da el soporte "científico" al artículo;

4.- Ios trabajos presentados en congresos no constituyen publicación, y para que un trabajo presentado en un congreso sea publicable, se deben hacer cambios de forma y fondo, en especial en lo que respecta a la documentación que da soporte al trabajo;

5.- los autores deben cuidar que sus escritos se ajusten a ciertas normas mínimas de forma y de fondo aceptadas por la comunidad internacional si desean que sus artículos, una vez publicados, tengan el impacto deseado; $y$

6.- el respeto que un autor o autora tenga por las Normas de una revista refleja su capacidad para ajustarse a ciertos requisitos y protocolos que son igualmente pedidos en los experimentos, en la formulación de modelos, en el desarrollo de software, en el análisis de encuestas, o simplemente en las reflexiones que se exponen en el artículo.

\section{REFERENCIAS}

Adare, A, y otros 410 autores, $\mathrm{J} / \psi$ Production versus Centrality, Transverse Momentum, and Rapidity in Au+Au Collisions at $\sqrt{ } s N N=200 G e V$, Phys. Rev. Lett. 98(23), 232301-232306 (2007).

Amin, M. y M. Mabe, Impact Factors: Use and Abuse, Elsevier Science Perspectives in Publishing., http://www1.elsevier.com/ homepage/ about/ita/editors/perspectives1.pdf (2000).

Bishop, C.T., How to Edit a Scientific Journal, ISI Press, Philadelphia, USA (1984).

Campanario, J.M., Cómo escribir y publicar un artículo científico. Cómo estudiar y aumentar su impacto, http://www2.uah.es/jmc/ (2009).

CIT, Centro de Información Tecnológica, La Serena-Chile, Normas y Documentos Varios http://www.citchile.cl/ a1a.htm (2010).

Day, R.A., How to Write and Publish a Scientific Paper, ISI Press, Philadelphia, USA (1983)

Packer, A.L., The SciELO Model for electronic publishing and measuring of usage and impact of Latin American and Caribbean scientific journals, $2^{\text {nd }}$ ICSU-UNESCO Int. Conference on Electronic Publishing in Science, UNESCO House, Paris (2003)

Plomp, R. The Highly Cited Papers of Professors as an Indicator of a Research Group's Scientific Performance. Scientometrics 29(3), 377-393 (1994).

Valderrama, J.O., La Publicación en Revistas Especializadas, un Recurso Estratégico en la Investigación Científica y Tecnológica. Cap.4 en Cellino, A. (editor), Los Laberintos del Futuro. Ciencia y Tecnología en América Latina, Edit. Univ. Nacional del Litoral, Santa Fe, Argentina (2001) Valderrama, J.O., "Principales Aspectos sobre la Preparación de un Artículo para ser Publicado en una Revista Internacional de Corriente Principal", Inf. Tecnol., 16(2), 03-14 (2005). 